\title{
Propriedades Psicométricas da Versão Brasileira da Escala de Satisfação das Necessidades Básicas no Esporte (BNSSS)
}

\author{
José Roberto Andrade do Nascimento Junior ${ }^{1,}$,, João Ricardo Nickening Vissoci ${ }^{2}$ \\ \& Lenamar Fiorese Vieira ${ }^{3}$ \\ ${ }^{1}$ Universidade Federal do Vale do São Francisco, Petrolina, PE, Brasil \\ ${ }^{2}$ Division of Emergency Medicine, Department of Surgery, Duke University Medical Center; Duke Global Neurosurgery \\ and Neurology, Department of Neurosurgery, Duke University Medical Center. Duke Global Health Institute, \\ Duke University, United States of America \\ ${ }^{3}$ Universidade Estadual de Maringá, Maringá, PR, Brasil
}

\begin{abstract}
RESUMO - Este estudo investigou as propriedades psicométricas da versão brasileira da Basic Needs Satisfaction in Sport Scale (BNSSS). O instrumento foi traduzido e adaptado por três especialistas da área. Um total de 475 atletas participaram deste estudo: 395 em um estudo de validade e $80 \mathrm{em}$ um estudo de estabilidade temporal. Os resultados demonstraram traduções claras e pertinentes entre os três especialistas, e consistência interna satisfatória dos itens em português. Diferentes modelos foram testados (AFE e AFC), sendo que o modelo mais estável sugeriu a exclusão de oito itens e retenção de três fatores. A análise multigrupos apontou a invariância parcial entre os sexos. A escala apresentou estabilidade temporal (CCI>0.70). Concluiu-se que a versão brasileira da BNSSS apresentou propriedades psicométricas aceitáveis.
\end{abstract}

PALAVRAS-CHAVE: psicometria, motivação, psicologia do esporte

\section{Psychometric Properties of the Brazilian Version of the Basic Needs Satisfaction in Sport Scale (BNSSS)}

\begin{abstract}
This study investigated the psychometric properties of the Brazilian version of the Basic Needs Satisfaction in Sport Scale (BNSSS). The instrument was translated and adapted by three experts. A total of 475 athletes participated in this study: 395 in a validity study and 80 in a temporal stability study. Factor analysis was used and reliability coefficients were estimated in the first study, while in the second study the intraclass correlation was calculated. The results showed clear and relevant translations between the three experts, and satisfactory internal consistency of items in Portuguese. Different models were tested (EFA and CFA), and the more stable model suggested the exclusion of eight items and the retention on three factors. Multigroup analysis showed partial invariance between the sexes. The scale showed temporal stability (ICC $>0.70$ ). It was concluded that the Brazilian version of BNSSS showed acceptable psychometric properties. KEYWORDS: psychometrics, motivation, sport psychology
\end{abstract}

Na última década, a teoria da autodeterminação(TAD) tem se solidificado como uma base teórica para a compreensão do comportamento e a motivação do ser humano para realização de tarefas durante seu desenvolvimento (Deci \& Ryan, 1985). Atualmente, sua aplicação vem se intensificando, com destaques para sua aplicabilidade em diferentes contextos, avaliados por diferentes procedimentos metodológicos (Deci \& Ryan, 2008; Vallerand, Pelletier \& Koestner, 2008). Esse desenvolvimento também é observado na área do esporte e do exercício. Estudo recente de revisão sistemática sobre o uso da TAD em pesquisas na área da educação física e do exercício revela que a teoria tem sido utilizada como referência em diversos países, analisando aspectos variados da experiência da atividade física (Teixeira, Carraça, Markland, Silva, \& Ryan, 2012). O mesmo acontece no Brasil, com uma emergência de estudos que utilizam o suporte da TAD (Vissoci, Vieira, Oliveira, \& Vieira, 2008; Silva, Matias, Viana \& Andrade, 2012).

Para a utilização da TAD em pesquisas com características psicométricas são necessários instrumentos de mensuração

*E-mail: jroberto.jrs01@gmail.com 
para seus constructos. No Brasil, na área do esporte já existem alguns instrumentos validados (Balbinotti \& Baerbosa, 2008; Bara Filho, Andrade, Miranda, Nuñez, Martín-Albó, \& Ribas, 2011) que avaliam alguns dos aspectos da TAD, como o continuum da autodeterminação e as regulações de motivação. No entanto, a micro-teoria do continuum da autodeterminação é uma das cinco microteorias que compõe a TAD autodeterminação (Deci \& Ryan, 2012). A micro-teoria das necessidades básicas tem sido utilizada como mediadora da relação entre diversas variáveis psicológicas no contexto esportivo e também como elemento influente em respostas e consequências, como motivação intrínseca, bem-estar, sensação de fluidez (Fernandes, Vasconcelos-Raposo, Laázaro \& Dósil, 2004; Reinboth, Duda, \& Ntoumanis, 2004; Adie, Duda, \& Ntoumanis, 2008). Nesse sentido, a lacuna se refere à Teoria das Necessidades Básicas, a qual vem aprofundando suas discussões nos últimos anos, apontando para a carência de instrumentos em língua portuguesa para a avaliação de tal variável no esporte.

De acordo com tal micro teoria, o indivíduo atinge a autodeterminação à medida que se sente satisfeito em relação às três necessidades psicológicas básicas: a) a autonomia, que se refere à percepção de que o comportamento e seus resultados não são influenciados por agentes externos, mas causados pelo próprio indivíduo; b) o senso de competência, determinado pela necessidade de se sentir efetivo e capaz de desempenhar atividades em níveis variados de dificuldade; e c) o senso de relacionamento, que é a necessidade de se sentir conectado a alguém ou ser alvo da preocupação de outras pessoas (Deci \& Ryan, 2012).

Em uma revisão da literatura, foram localizadas três escalas que se propõem a avaliar as necessidades básicas relacionadas à atividade física: a Psychological Need Satisfaction in Exercise Scale-PNSE (Wilson, Rogers, Rodgers, \& Wild, 2006) e a Basic Psychological Needs in Exercise Scale-BPNES (Vlachopoulos \& Michailidou, 2006) - desenvolvidas no mesmo período para o contexto do exercício físico - e a Échelle de Satisfaction des Besoins Psychologiques (Gillet, Rosnet, \& Vallerand, 2008).

Embora existam instrumentos para avaliar a satisfação de necessidades básicas, o primeiro instrumento desenvolvido especificamente para o esporte foi a Basic Needs Satisfaction in Sport Scale (Ng, Lonsdale, \& Hodge, 2011). Na língua portuguesa, por sua vez, iniciativas de estudar as necessidades básicas no esporte têm se efetuado por meio de instrumentos adaptados de outros contextos ou avaliando necessidades específicas (Moutão, Cid, Leitão, \& Alves, 2012). Contudo, ainda são escassos os estudos que utilizam essa perspectiva teórica, sendo que um dos motivos pode ser a falta de instrumentos adequados e validados para o contexto esportivo brasileiro.

Diante disso, o presente estudo teve como objetivo adaptar e analisar as propriedades psicométricas para o contexto esportivo brasileiro a Basic Needs Satisfaction in Sport Scale (BNSSS), buscando especificamente avaliar a validade de conteúdo, a validade de constructo (consistência interna e estrutura fatorial) e a estabilidade temporal do instrumento.

\section{MÉTODOS}

\section{Participantes}

A amostra de validação foi composta por 395 atletas adultos das modalidades coletivas e individuais (186 do sexo feminino e 209 do sexo masculino) da fase final dos Jogos Abertos do Paraná (JAPS) - 2012, oriundos de diferentes regiões do Brasil com idade média 22.53 \pm 5.92 anos. O tamanho da amostra foi determinado com base nas recomendações da literatura (Hair, Black, Babin, Anderson, \& Tatham, 2009; Marôco, 2010), que propõem o mínimo 10 sujeitos por item e parâmetro a ser estimado para realização da Análise Fatorial Exploratória e Confirmatória, respectivamente. Os sujeitos praticavam as seguintes modalidades: ginástica rítmica (10), taekwondo (42), vôlei de praia (38), ciclismo (12), natação (43), judô (38), atletismo (33), tênis (10), rúgbi (15), handebol (75), vôlei (32), basquete (23) e futsal (24).

Para a análise da estabilidade temporal, foram selecionados de forma não probabilística, por conveniência, uma amostra independente de 80 atletas de diferentes modalidades esportivas (futsal, natação, atletismo e voleibol) do estado do Paraná, os quais responderam a versão validada da BNSSS em dois momentos distintos (teste e reteste) com intervalo de sete dias após a testagem inicial (Nunnally \& Berstein, 1994).

Os critérios de inclusão da amostra foram: 1) ter participado de alguma competição de nível nacional durante a temporada 2012 ; 2) ter participado do Campeonato Estadual da Modalidade durante a temporada 2011/2012; e 3) estar classificado para a fase final dos JAPS 2012, representando assim, atletas e equipes de alto rendimento. Todos os participantes (tradutores, doutores e atletas) consentiram em participar do estudo por meio do Termo de Consentimento Livre e Esclarecido (TCLE).

\section{Instrumento}

A escala original utilizada foi a Basic Needs Satisfaction in Sport Scale (BNSSS) de Ng et al. (2011). Tal instrumento avalia as percepções dos atletas em relação a sua competência, autonomia e relacionamento no contexto esportivo. A escala é constituída por 20 itens distribuídos em cinco subescalas: competência (Itens 6, 11, 12, 14 e 17); autonomia para a escolha (Itens 4, 9, 13 e 20); autonomia para a vontade (Itens 2, 15 e 16); autonomia para o lócus de causalidade 
interna percebida (Itens $3,5^{*}$ [pontuação inversa] e 8); e relacionamento (Itens 1, 7, 10, 18 e 19). De acordo com Ng et al. (2011), as três subescalas de autonomia (escolha, lócus de causalidade interna percebida e vontade) podem gerar uma pontuação geral por meio da média das pontuações das três subescalas. As respostas são dadas numa escala tipo Likert de sete pontos, num continuum de "Não é totalmente verdade" (1) a "Totalmente verdade" (7). O escore de cada subescala é calculado a partir da média do somatório dos itens que a compõem. Valores mais elevados nas dimensões descritas significam maior satisfação das necessidades psicológicas básicas.

\section{Tradução do Instrumento}

O instrumento foi adaptado através do método de tradução dupla (reversa e independente - back translation). Primeiramente dois tradutores de língua materna portuguesa, contratados de forma independente, traduziram para o português a BNSSS; em um segundo momento, dois outros tradutores de língua nativa inglesa, converteram para o inglês as versões traduzidas para o português. As versões em língua portuguesa foram unificadas, resultando na versão final do instrumento (Pasquali, 2010) através de um painel de pesquisadores e também, de discussão e consenso. Esse painel foi composto por professores doutores não participantes do estudo, contatados por e-mail ou pessoalmente. Entre as atividades do painel, realizou-se correções dos termos técnicos utilizados na tradução e a adequação dos itens foram realizadas. Discordâncias foram sanadas pelos autores do estudo. $\mathrm{O}$ instrumento traduzido para o português foi intitulado Escala de Satisfação das Necessidades Básicas no Esporte (BNSSS).

Cinco professores universitários doutores na área de Psicologia do Esporte foram convidados a participar como juízes avaliadores, para verificar a adequação dos itens da versão original da BNSSS, respondendo a escala de clareza e pertinência. A análise teórica dos 20 itens da BNSSS foi realizada por meio da técnica para avaliação da validade de conteúdo apresentada por Hernández-Nieto (2002). Utilizouse a escala de clareza de linguagem e pertinência prática do tipo Likert (em cinco pontos), a qual foi respondida pelos juízes avaliadores a fim de verificar a validade de conteúdo da escala. Terminado o processo de adaptação, foi realizado um estudo piloto com a versão final do instrumento, que foi aplicado a 20 atletas de diferentes modalidades esportivas. Não houve a necessidade de alteração no conteúdo do instrumento adaptado após a aplicação na amostra piloto.

Após a finalização do processo de validação de conteúdo, foi realizado um estudo piloto com um grupo de 20 atletas, selecionados por conveniência e estratificados por sexo, com o objetivo de avaliar as questões do instrumento quanto à linguagem e conteúdo (Marôco, 2010). Para o cálculo do coeficiente de validade de conteúdo, utilizou-se a técnica proposta por Hernandez-Nieto (2002), com ponto de corte de 0.80 . Tal análise foi realizada por meio do coeficiente de validade de conteúdo para cada item do instrumento (CVCc) e para o questionário como um todo $(\mathrm{CVCt})$. Também foi realizado o cálculo do coeficiente Kappa, que indica o grau de concordância entre os juízes quanto ao pertencimento de cada item à sua respectiva dimensão.

\section{Procedimentos}

O estudo foi aprovado pelo Comitê Permanente de Ética em Pesquisa com Seres Humanos da Universidade (Parecer $\mathrm{n}^{\circ} 131.681 / 2012$ ). Inicialmente, solicitou-se a autorização formal para a tradução e adaptação transcultural da BNSSS ao Professor PhD Chris Lonsdale, autor do instrumento original na Austrália.

Inicialmente foi realizado contato com a Secretaria de Esporte do Estado do Paraná (entidade organizadora dos JAPS), a fim de obter autorização para a coleta de dados da pesquisa com os atletas e as equipes participantes da competição. A aplicação da BNSSS foi realizada nos alojamentos dos atletas na cidade onde ocorreu a competição, no segundo semestre de 2012. Procedimento semelhante foi executado em relação à amostra selecionada para a análise da estabilidade temporal do instrumento (teste e reteste), com duas aplicações num intervalo de sete dias, nos locais de treinamento dos atletas de acordo com horário pré-determinados pela comissão técnica no final do primeiro semestre de 2013.

\section{Análise dos Dados}

Os dados relativos à caracterização da amostra foram analisados de forma descritiva (média e desvio padrão), para dados contínuos, e percentual, para os dados categóricos.

Para a análise da validade de constructo da versão brasileira da BNSSS, avaliamos a confiabilidade dos itens através do Alfa de Cronbach e confiabilidade composta (consistência interna). Como se tratara da primeira tradução e adaptação do instrumento para a realidade brasileira, procuramos estudar as propriedades psicométricas da BNSSS com abordagens convergentes, visando corroborar os resultados por diferentes métodos. Dessa forma, empregamos inicialmente a avaliação de conteúdo pelos jurados, junto ao processo de tradução e adaptação do instrumento. Em sequência, aplicamos a Análise Fatorial Exploratória/AFE para verificar como os itens se comportariam a partir da estrutura dos dados. Por fim, testamos o modelo conceitual proposto pelo estudo de criação do BNSSS com os dados da amostra brasileira através da Análise Fatorial Confirmatória/ AFC, analisando a variância extraída média para verificar a validade convergente $\mathrm{e}$ a análise multigrupos para avaliar a invariância entre homens e mulheres (Hair et al., 2009; Marôco, 2010; Kline, 2012).

Na AFE, o coeficiente KMO $(0,87)$ e a análise de esfericidade de Bartlett $(\mathrm{p}=0,001)$ garantiram a confiabilidade do modelo exploratório. Foi utilizado o método de extração dos Mínimos Quadrados Não- 
Ponderados, uma vez que o mesmo suporta que os itens não tenham distribuição normal, com rotação Oblíqua Direta. A análise da medida de adequação da amostra de cada item foi realizada por meio da matriz de anti-imagem (MAS $>0,70 \mathrm{e}$ $\mathrm{r}<0,09)$. Cargas fatoriais acima de 0,50 foram consideradas aceitáveis (Hair et al., 2009).

AFC foi conduzida inicialmente através de uma análise preliminar a fim de constatar a adequação dos dados à análise. Trata-se precisamente da verificação da existência de casos aberrantes (outliers), visto que a inexistência desses casos é um pressuposto para esta análise. Os outliers foram avaliados por meio da distância de Mahanalobis, a qual não evidenciou a existência dos mesmos, permitindo o uso da AFC. Verificouse também a normalidade, tendo-se estudado não só a distribuição univariada dos dados por meio da assimetria $(S k)$ e da curtose $(K u)$, mas também a distribuição multivariada (coeficiente de Mardia para a curtose multivariada) (ISkI< 3,0 e $I K u I<10,0$ ) (Hair et al., 2009).

A adequação do modelo fatorial confirmatório foi testada com o uso do método de estimação de Máxima verossimilhança, que é o mais indicado em grandes amostras como a do presente estudo (Marôco, 2010). Para verificar a estabilidade e significância da carga fatorial $(>0,50)$ de cada item com seu respectivo fator, foi utilizada a técnica de Bootstrapping (Cheung \& Lau, 2008). O modelo final da BNSSS foi testado por meio dos índices de ajuste mais recomendados na literatura (Hair et al., 2009; Kline, 2012): Qui-Quadrado ( $X^{2}$ e p-valor), Índice de Qualidade do Ajuste (GFI > 0,90), Raiz do Erro Quadrático Médio de Aproximação (RMSEA < 0,08, I.C. 90\%, p-value [H0: RMSEA $\leq 0,05]$ ), Índice de Ajuste Normalizado (NFI $>0,90$ ), Índice Tucker-Lewis (TLI > 0,90), Índice de Qualidade de Ajuste Calibrado (AGFI > 0,90), Qui-Quadrado Normalizado ( $\mathrm{X}^{2} /$ grau de liberdade, recomendado entre 1,0 e 3,0) e Índice de Ajuste Comparativo (CFI > 0,90).

Para avaliar a validade convergente, empregou-se a análise da Variância Extraída Média (VEM), conforme sugerido por Fornell \& Larcker (1981), e valores próximos ou superiores a 0,50 foram considerados indicadores de adequada validade convergente. Também foi calculada a Confiabilidade Composta (CC) por meio dos resultados da $\mathrm{AFC}$, sendo que valores superiores a 0,70 foram considerados indicadores de adequada CC (Hair et al., 2009).

Para a identificação da invariância fatorial do instrumento, inicialmente definiu-se um modelo de adequação apropriado para cada sexo. Após a obtenção dos fatores, estes foram submetidos simultaneamente a uma análise multi grupos (com correção Emulisrel6) que visou efetuar um conjunto progressivo de restrições (cargas fatoriais, variâncias e covariâncias), de forma a analisar a equivalência do instrumento para diferentes subgrupos (homens vs mulheres).

Para a análise da estabilidade temporal da escala efetuou-se o Coeficiente de Correlação Intraclasses (CCI), verificando-se assim a fidedignidade teste-reteste do instrumento. Foi adotado para o presente estudo o índice mínimo de 0,70 (Nunnally \& Berstein, 1994). Todas as análises foram conduzidas por meio dos softwares SPSS versão 19,0 e Amos versão 17,0.

\section{RESULTADOS}

\section{Análise Teórica dos Itens (validade de conteúdo da BNSSS)}

Verificou-se que todas as dimensões da BNSSS obtiveram coeficientes de validade de conteúdo (CVC) em relação à clareza de linguagem e à pertinência prática acima de 0.80 , com exceção da dimensão autonomia para o lócus de causalidade interna percebida, que apresentou CVC para pertinência prática de 0,66 , indicando que essa dimensão não possui pertinência prática satisfatória. Além disso, os juízes avaliadores afirmaram que os itens relacionados à temática autonomia (autonomia para a escolha, autonomia para a vontade, autonomia para o lócus de causalidade interna percebida) não eram facilmente diferenciáveis. Razão pela qual recomendaram que fossem unidos para se criar apenas uma dimensão geral de autonomia.

Assim, para a análise da dimensão teórica, por sugestão do painel de avaliadores, o modelo sofreu mudanças em relação ao instrumento original, sendo considerada apenas uma dimensão geral de autonomia. Dessa forma, as distribuições dos itens em três dimensões da escala foram alterados: 1) competência (itens $6,11,12,14,17) ; 2$ ) autonomia (itens $2,3,4,5^{*}$ pontuação inversa, $8,9,13,15,16,20$ ); e 3 ) relacionamento (itens $1,7,10,18,19$ ). A classificação dos itens nas subescalas competência, autonomia e relacionamento apresentaram forte concordância com valor de Kappa de 0,84. Apesar das mudanças recomendadas pelos juízes avaliadores e da forte concordância com três dimensões (autonomia, competência e relacionamento), para a análise da estrutura fatorial (AFE e AFC), testamos diferentes modelos com o intuito de encontrar o modelo com melhor ajuste, uma vez que inconsistências foram encontradas na análise de conteúdo.

\section{Estatística Descritiva e Consistência Interna da BNSSS}

A análise revelou que os atletas recorreram às sete possibilidades de resposta existentes para cada um dos 20 itens da BNSSS. As médias das respostas situaram-se entre 4,86 $\pm 1,84$ e $6,25 \pm 1,21$, respectivamente, nos itens 5 ("Em meu esporte, me sinto forçado a fazer coisas que 
não gostaria de fazer") e 8 ("Eu pratico meu esporte por vontade própria"). $\mathrm{O}$ índice de consistência interna geral da BNSSS foi de 0,85 . Verificou-se que o alfa de Cronbach das dimensões da escala foi satisfatório $(\alpha=0,83$ a $\alpha=0,86)$. Nota-se, também, a existência de correlações item-dimensão que variaram de $0,50 \leq r \leq 0,83$, indicando correlação moderada e forte entre as dimensões e seus respectivos itens.

\section{AFE da BNSSS}

A AFE foi efetuada com o objetivo de verificar como os itens da escala da versão brasileira da BNSSS se comportariam nas respectivas dimensões. A análise da matriz de correlação entre os itens também sugeriu que eles poderiam ser explicados por fatores latentes, uma vez que apresentaram correlações significativas e acima de 0,30 com os itens das mesmas dimensões, com exceção do item 5 que apresentou correlação abaixo de 0,20 com todos os demais. Contudo, analisando a matriz de correlação também percebeu-se que os itens $1,2,4,7,9,13,15,16$ e 20 apresentavam equiparações fortes $(r>0,40)$ com itens de outras dimensões. Percebe-se que a maioria destes itens $(2,4$, $9,13,15,16,20)$ era pertencente às dimensões de Autonomia (Escolha, Vontade e Lócus de Causalidade), o que revelou indícios de inconsistências do modelo de cinco fatores.

Apesar da validação de conteúdo da escala sugerir um modelo de três fatores, incialmente foi testado um modelo com a extração livre de fatores afim de verificar como os itens se distribuiriam pelas dimensões. Apesar da análise dos eigenvalues pelo critério de $\operatorname{Kaiser}(>1,0)$ sugerir a retenção de apenas três fatores, a análise paralela e o critério de Cattel (scree plot) não deixaram totalmente claro esta extração. Dessa forma, optamos por testar três modelos exploratórios (três, quatro e cinco fatores), como uma medida de sensibilidade da estrutura fatorial dos itens.

Inicialmente, foi testado o modelo de cinco fatores (seguindo o modelo original do instrumento teorizando dimensões para Competência, Relacionamento, Autonomia para Escolha, Vontade e Lócus de Causalidade), o qual explicou 59\% da variância dos dados. No entanto, verificouse que o fator 1 foi saturado por itens que seriam pertencentes a todas dimensões da escala, indicando uma instabilidade no modelo de cinco fatores. Especificamente, observou-se que todos os itens $(3,5$ e 8$)$ do fator Autonomia para o Lócus de Causalidade, o item 2 (Vontade), 6 (Competência) e 10 (Relacionamento) apresentaram carga fatorial cruzada com os outros fatores de autonomia.

No modelo de quatro fatores, que explicou $55 \%$ da variância dos dados, foi encontrado o mesmo problema de carga fatorial cruzada entre os itens (1, 4, 6 e 10), além do que os itens $5(\mathrm{CF}=0,23), 6(\mathrm{CF}=0,35), 7(\mathrm{CF}=0,31)$ e 10 $(\mathrm{CF}=0,28)$ não saturaram com $\mathrm{CF}>0,50$ em seus respectivos fatores. Um dos fatores (relacionamento) extraiu apenas dois itens com $\mathrm{CF}>0,50$, tornando inconsistente o modelo de quatro fatores. Dessa forma, foi testado um modelo de três fatores (Autonomia, Competência e Relacionamento), o qual apresentou melhor ajuste e distribuição dos itens pelos fatores (cada fator apresentou pelo menos três itens semelhantes do instrumento original com $\mathrm{CF}>0,50$ ), explicando $48,8 \%$ da variância total dos dados. Contudo, este modelo também apresentou itens $(1,4,6,7,9,13 \mathrm{e}$ 20) com cargas fatoriais cruzadas acima de $0,30 \mathrm{em}$ fatores diferentes do modelo original e o item 5 saturou com $\mathrm{CF}<0,50 \mathrm{em}$ seu respectivo fator. Apesar de ter apresentado oito itens problemáticos, o modelo de três fatores foi o mais estável, visto que foram observadas maiores cargas fatoriais e comunalidades dos itens.

A partir destes resultados exploratórios, optamos por testar um quarto modelo sem os oito itens problemáticos $(1,4,5,6,7,9,13$ e 20$)$ do modelo de três fatores (Tabela 1). Este modelo apresentou adequado ajuste $(\mathrm{KMO}=0,85$ / Bartlett $\mathrm{p}<0,001$ ) e todos os indicadores para a quantidade de fatores a extrair concordaram em três dimensões, explicando $61 \%$ da variância total dos dados.

Tabela 1. AFE do modelo de 3 fatores da versão brasileira da BNSSS.

\begin{tabular}{|c|c|c|c|}
\hline Itens da BNSSS & Autonomia & Relacionamento & Competência \\
\hline 2. Em meu esporte, sinto que estou perseguindo meus próprios objetivos & 0,63 & & \\
\hline 3. Eu sinto que pratico meu esporte por prazer & 0,69 & & \\
\hline 8. Eu pratico meu esporte por vontade própria & 0,64 & & \\
\hline 10. Existem pessoas em meu esporte que se preocupam comigo & & 0,55 & \\
\hline 11. Eu sou habilidoso em meu esporte & & & 0,83 \\
\hline 12. Eu sinto que sou bom em meu esporte & & & 0,80 \\
\hline 14. Eu tenho oportunidade de sentir que sou bom em meu esporte & & & 0,67 \\
\hline 15. Em meu esporte, eu realmente tenho a sensação de querer estar nele & 0,63 & & \\
\hline 16. Em meu esporte, sinto que estou fazendo o que eu quero fazer & 0,60 & & \\
\hline 17. Eu tenho habilidade para ter um bom desempenho em meu esporte & & & 0,58 \\
\hline 18. Em meu esporte, existem pessoas em quem eu posso confiar & & 0,56 & \\
\hline 19. Eu tenho um relacionamento próximo com as pessoas que praticam meu esporte & & 0,49 & \\
\hline Eigenvalue & 6,10 & 2,21 & 1,47 \\
\hline Percentual de Variância Explicada & 30,47 & 11,08 & 7,34 \\
\hline
\end{tabular}

Nota: Método de Extração dos Mínimos Quadrados Não-Ponderados. 
Verificou-se também que (Tabela 1) todos os itens saturaram com $\mathrm{CF}>0,50$ em seus respectivos fatores: Autonomia (Itens 2, 3, 8, 15 e 16), Competência (11, 12,14 e 17) e Relacionamento (10, 18 e 19). Tal achado aponta indicativos para uma estrutura mais estável da versão brasileira da BNSSS com três fatores (Autonomia, Competência e Relacionamento), entretanto, optamos por testar diferentes modelos na AFC.

\section{AFC da BNSSS}

Inicialmente foi testado um modelo de cinco fatores com os 20 itens (M1) para analisar como eles se comportariam em seus respectivos fatores e para testar um modelo com a mesma estrutura do original. Percebeu-se que os mesmos oito itens problemáticos da $\operatorname{AFE}(1,4,5,6,7,9,13$ e 20) apresentaram carga fatorial $<0.50$ na análise preliminar da AFC, sendo a maioria deles pertencentes às dimensões de autonomia (Escolha, Vontade e Lócus de Causalidade). O modelo (M1) apresentou ajuste inadequado $\left[\mathrm{X}^{2}(160)=612,89 ; \mathrm{X}^{2} / \mathrm{gl}=\right.$ 3,$83 ; \mathrm{CFI}=0,83$; AGFI $=0,74 ; \mathrm{GFI}=0,85 ; \mathrm{TLI}=0,79$; RMSEA $=0,09(0,08-0,10)$; $\mathrm{P}(\mathrm{RMSEA}<0,05)=0,001]$. Outro modelo testado (M2) foi um modelo de cinco fatores de $1^{\text {a }}$ ordem (semelhante ao original) com a postulação de um fator de $2^{\mathrm{a}}$ ordem (Autonomia) a partir das três dimensões de $1^{\text {a }}$ ordem (Escolha, Vontade e Lócus de Causalidade). Contudo, tal modelo apresentou ajuste ainda mais pobre $\left[\mathrm{X}^{2}(164)=710,33 ; \mathrm{X}^{2} / \mathrm{gl}=4,333 ; \mathrm{CFI}=0,80 ; \mathrm{GFI}=0,83\right.$; $\mathrm{TLI}=0,75$; RMSEA $=0,09(0,08-0,10) ; \mathrm{P}(\mathrm{RMSEA}<0,05)$ $=0,001]$ e revelou as mesmas inconsistências em relação às $\mathrm{CF}$ dos oito itens do modelo anterior.

Além disso, testamos três modelos avulsos para as variáveis latentes de autonomia (Escolha, Vontade e Lócus de Causalidade) com o intuito de verificar se tais fatores apresentavam estrutura aceitável individualmente, porém nenhum dos três modelos apresentou ajuste aceitável, além do que os itens $2,3,5,8$ e 10 não saturaram com $\mathrm{CF}>0.50$ em seus respectivos fatores.

A partir de todos os indicativos dos modelos testados anteriormente, optou-se por testar um modelo (M3) com os 20 itens, postulando a existência de três fatores (Autonomia, Competência e Relacionamento), com o intuito de verificar como os itens problemáticos carregariam em uma dimensão única de autonomia. O M3 também apresentou ajuste insatisfatório $\left[\mathrm{X}^{2}(167)=871,25 ; \mathrm{X}^{2} / \mathrm{gl}=5,217 ; \mathrm{CFI}=\right.$ 0,$73 ; \mathrm{GFI}=0,80 ; \mathrm{TLI}=0,70 ;$ RMSEA $=0,10(0,09-0,11)$; $\mathrm{P}(\mathrm{RMSEA}<0,05)=0,001]$ e revelou as mesmas inconsistências em relação às $\mathrm{CF}$ dos oito itens do modelo anterior.

Diante dos achados anteriores, testamos um modelo (M4) de três fatores sem os oito itens problemáticos. Assim, o M4 foi composto por 12 itens, sendo que todos eles saturaram nos três fatores com uma magnitude superior a $0,50(p<0,001)$. Ao analisar a estrutura fatorial do M4, relativamente ao ajustamento global do modelo, verificouse que a amostra avaliada apresentou um $\mathrm{X}^{2}=189,46 \mathrm{e}$ significativo para $\mathrm{p}<0,001$, sugerindo um fraco ajuste. As demais medidas de ajuste absoluto obtiveram valores aceitáveis pela literatura (GFI $=0,93$; RMSEA $=0,08, \mathrm{I}, \mathrm{C}$, $0,07-0,10)$, Todas as medidas de ajuste incremental (TLI = $0,89$; AGFI $=0,90)$ atingiram ou estivem muito próximas do nível recomendado, suportando a aceitação do modelo proposto. Quanto às medidas de ajuste parcimonioso, o QuiQuadrado normalizado $\left(\mathrm{X}^{2} / \mathrm{gl}=3,18\right)$ pode ser considerado aceitável e o CFI $(0,91)$ alcançou o nível recomendado. Neste modelo (M4), os Índices de Modificação recomendaram uma correlação entre os erros dos itens 3 e $8(0,25)$, ambos do fator autonomia.

A Figura 1 apresenta as estimativas do modelo final da BNSSS com 12 itens (M4), o qual descreve também a relação entre as dimensões e os indicadores na solução encontrada para os dados de validação da BNSSS. As saturações fatoriais $(\lambda)$ apresentaram valores moderados e fortes situados entre 0,51 e 0,84 . As replicações bootstrap $(\mathrm{p}<0,001)$ e Intervalo de Confiança (I.C. 90\%) indicaram a estabilidade das estimações fatoriais e o ajuste do modelo para os dados.

Os valores da VEM foram os seguintes: Competência $=$ 0,61 ; Autonomia $=0,48$; Relacionamento $=0,47$. Os fatores Autonomia e Relacionamento apresentaram uma validade convergente abaixo do limite proposto, entretanto, próxima do valor recomendado (VEM $>0,50)$. Os valores da $\mathrm{CC}$ foram satisfatórios para avaliação da consistência interna (Competência $=0,82 ;$ Autonomia $=0,78$; Relacionamento $=0,70)$.

\section{Invariância da BNSSS entre Homens e Mulheres}

Ao analisar a invariância configuracional do modelo modificado da BNSSS com 12 itens (M4) entre homens e mulheres $\left[\mathrm{X}^{2}(98)=272,19 ; \mathrm{X}^{2} / \mathrm{gl}=2,77 ; \mathrm{CFI}=0,90\right.$; $\mathrm{GFI}=0,90 ; \mathrm{TLI}=0,89 ;$ RMSEA $=0,067(0,056-0,082)$; $\mathrm{P}(\mathrm{RMSEA}<0,05)=0,002]$, observou-se que o modelo apresentou um ajuste adequado entre atletas do sexo masculino e do sexo feminino, indicando que a estrutura proposta mantém-se estável nos dois grupos. Verificou-se que o modelo constrito com pesos fatoriais em atletas (homens $\mathrm{x}$ mulheres) não apresentou um ajuste significativamente pior do que o modelo com os parâmetros livres $\left[\mathrm{X}^{2} \operatorname{diff}(9)=\right.$ $13,79 ; \mathrm{p}=0,08]$. Tal achado evidencia que o modelo com pesos fatoriais fixos possui bom ajuste para ambos os grupos assim como o modelo com parâmetros livres, demostrando a equivalência métrica dos pesos de medida entre homens e mulheres. No entanto, a invariância estrutural do modelo não foi obtida [ $\left.\mathrm{X}^{2} \operatorname{diff}(15)=35,86 ; \mathrm{p}=0,01\right]$, demonstrando que os níveis de correlação entre os fatores não são os mesmos para ambos os grupos. Tais resultados demonstram a invariância parcial (configuracional e métrica) do modelo modificado da BNSSS com 12 itens em ambos os grupos.

Finalmente, a numeração e a reordenação dos 12 itens pelas três dimensões foram corrigidas para a finalização do processo de validação da BNSSS, destacando que a estrutura da versão para a língua portuguesa se alterou em relação ao modelo original (Ng et al., 2011). Dessa forma, a 


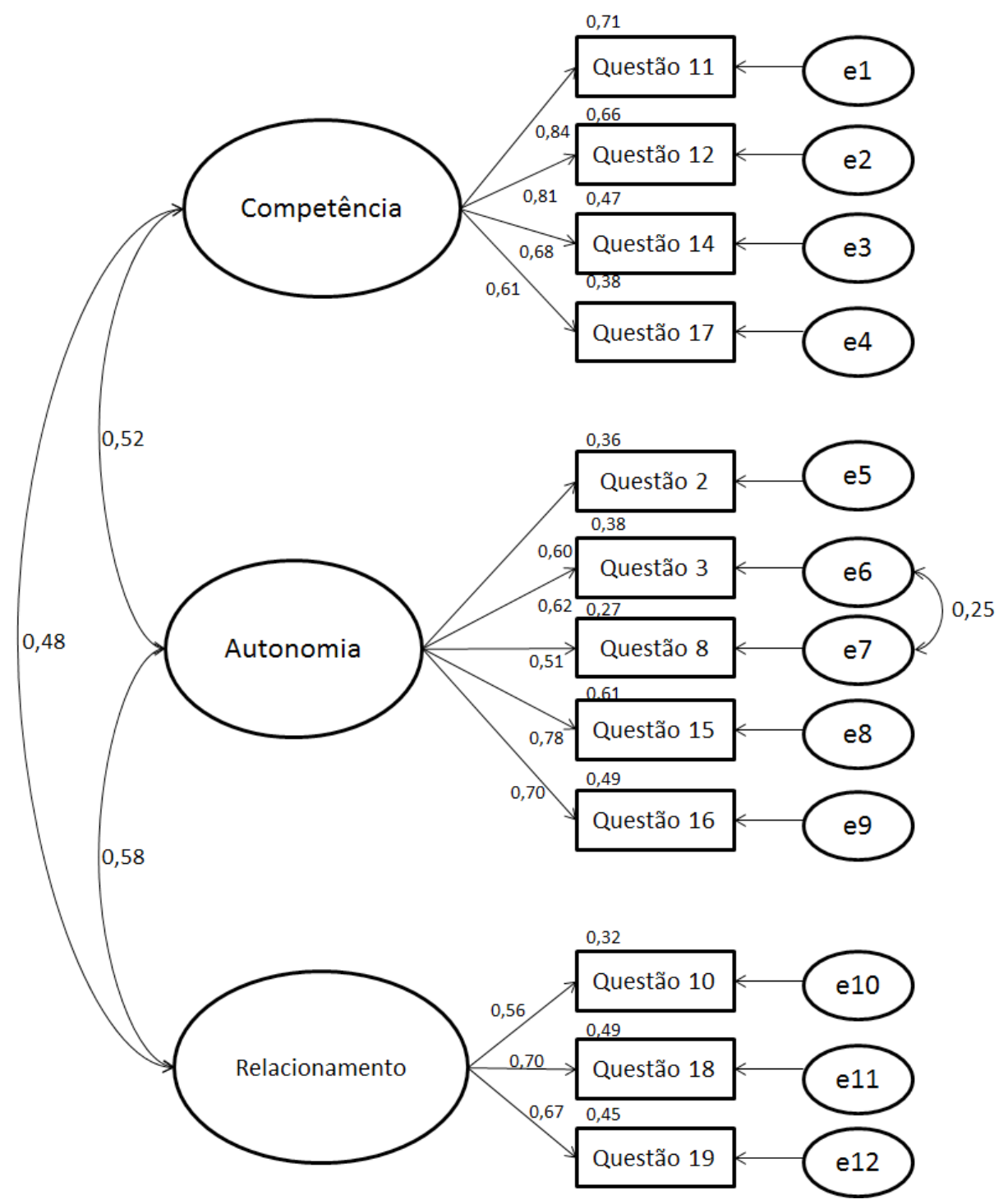

Figura 1. Carga fatorial, intercorrelações entre fatores e erro associado a cada item no modelo de 3 fatores com 12 itens da BNSSS (M4).

BNSSS ficou com a seguinte distribuição: 1) Competência (Itens 5, 6, 7 e 10); 2) Autonomia (Itens 1, 2, 3, 8 e 9); e 3 ) Relacionamento (Itens 4, 11 e 12).

\section{Estabilidade Temporal da BNSSS}

Os valores dos CCI apontaram a confirmação da fidedignidade (estabilidade temporal) da escala. Todos os itens encontram-se acima do índice mínimo recomendado pela literatura $(r>0,75)$. Ao agrupar os 12 itens, encontrouse correlação intraclasse média de 0,79 , evidenciando a estabilidade temporal dos itens da escala. Por se tratar de um instrumento multidimensional, verificou-se que todas as dimensões da BNSSS apresentaram CCI entre $r=0,76 \mathrm{e}$ $r=0,81$, o que indica uma forte fidedignidade entre teste $\mathrm{e}$ reteste para a amostra de validação.

\section{DISCUSSÃO}

Este é o primeiro estudo de tradução e adaptação da escala de necessidades básicas específica para o contexto esportivo brasileiro, e de verificação de suas propriedades psicométricas. A versão brasileira da BNSSS apresentou resultados aceitáveis nas análises de conteúdo, confiabilidade, validade fatorial exploratória e confirmatória e estabilidade temporal, evidenciando que a avaliação das necessidades psicológicas básicas no contexto esportivo por meio da BNSSS pode ser efetuada. No entanto, é importante destacar que a estrutura final do teste compreendeu três construtos (autonomia, competência e realização) e 12 itens, diferenciando-se da escala original que subdivide a autonomia em três subdimensões e possui 20 itens. 
De maneira geral, a versão brasileira da BNSSS apresentou indicadores satisfatórios de consistência interna e validade de construto. Como os autores do instrumento original (Ng et al., 2011) afirmam que o procedimento de juntar as dimensões de autonomia é passível de ser realizado, optamos por atender as sugestões dos juízes avaliadores e unir as três dimensões de autonomia em uma dimensão geral. Tal evidência também foi comprovada pelos resultados da AFE, que apontou a retenção de apenas três fatores.

Dado que a versão brasileira é a primeira tradução e adaptação transcultural da BNSSS reportada, não temos como comparar o resultado da análise de conteúdo com o de outras culturas. Entretanto, nota-se que a divisão da dimensão de autonomia em subdimensões é um aspecto reportado na literatura (Reeve, Nix, \& Hamm, 2003; Ng et al., 2011), mas não representa um consenso entre os pesquisadores. A maior parte das escalas que avaliam as necessidades psicológicas básicas no esporte ou exercício físico estrutura-se em três dimensões (Vlachopoulos \& Michailidou, 2006; Gillet et al, 2008; Domínguez et al., 2010), como originalmente constituída no modelo teórico da TAD (Deci \& Ryan, 2012).

Nos dados analisados, constatou-se que dos 20 itens inicialmente incluídos no modelo de AFE, oito apresentaram problemas. O mesmo tipo de resultado foi verificado na versão original da escala. Contudo, a exclusão desses itens não interferiu nos indicadores de adequação do modelo; portanto, foram mantidos (Ng et al., 2011). No caso do presente estudo, verificou-se que os itens influenciavam a estrutura fatorial, razão pela qual foram excluídos. Todos esses itens eram de outras dimensões, mas acabaram por carregar com maior magnitude na dimensão Autonomia.
O mesmo problema foi encontrado com a saturação dos quatro itens que faziam parte da subdimensão choice (escolha) da versão original da BNSSS. Apesar da preferência na literatura por uma subdivisão da autonomia (Hodge, Lonsdale \& Ng, 2008), a subdimensão choice apresentou correlações mais fracas em critérios de validação de conteúdo na versão original (Ng et al., 2011), não se comportando conforme a hipótese do estudo. Isso evidenciou que o construto da autonomia apresentava dissonâncias quanto a suas ramificações, necessitando mais investigações. Dessa forma, associando as cargas fatoriais dos itens $(4,9,13$ e 20), as considerações dos juízes na validação de conteúdo e as discussões da literatura, optou-se por manter apenas um construto para a autonomia, excluindo os itens problemáticos.

Apesar da remoção de 8 itens, o modelo da versão brasileira da BNSSS (Tabela 2) apresentou indicadores de adequação aceitáveis na AFC (Hair et al., 2009; Kline, 2012), revelando coerência teórica em relação ao modelo proposto no questionário original. Os índices de ajuste também se mostraram satisfatórios com relação aos atletas da amostra de validação, comprovando a validade de constructo do instrumento para o contexto brasileiro, corroborando os achados do instrumento para outras culturas (Vlachopoulos \& Michailidou, 2006; Wilson et al., 2006; Gillet et al., 2008; Domínguez et al., 2010). Embora os resultados da AFC suportem a validade convergente do modelo, verificou-se a existência de correlação entre os erros dos itens 3 e 8 (Figura 1). No entanto, essa relação entre os erros desses itens foi fraca $(<0,40)$ e entre itens do mesmo fator, o que não afeta a validade fatorial do modelo da BNSSS, visto que não pode ser considerada como um indicador de cross-loading entre os itens e as dimensões do instrumento (Hair et al., 2009; Marôco, 2010).

Tabela 2. Índices de ajustamento dos modelos da versão brasileira da BNSSS testados pela AFC.

\begin{tabular}{|c|c|c|c|c|}
\hline $\begin{array}{l}\text { Comparação entre os } \\
\text { modelos da BNSSS }\end{array}$ & $\begin{array}{c}\text { Modelo de } 5 \text { fatores com } 20 \\
\text { itens (M1) }\end{array}$ & $\begin{array}{l}\text { Modelo de } 5 \text { fatores (20 } \\
\text { itens) com } 2^{\mathrm{a}} \text { ordem (M2) }\end{array}$ & $\begin{array}{c}\text { Modelo de } 3 \text { fatores com } \\
20 \text { itens (M3) }\end{array}$ & $\begin{array}{c}\text { Modelo de } 3 \text { fatores com } 12 \\
\text { itens (M4) }\end{array}$ \\
\hline $\mathrm{X}^{2}$ & 612,89 & 710,33 & 871,25 & 189,46 \\
\hline $\mathrm{gl}$ & 160 & 164 & 167 & 50 \\
\hline p-valor & 0,001 & 0,001 & 0,001 & 0,001 \\
\hline$X^{2}$ normalizado $\left(X^{2} / \mathrm{gl}\right)$ & 3,83 & 4,33 & 5,21 & 3,18 \\
\hline GFI & 0,85 & 0,83 & 0,80 & 0,93 \\
\hline $\begin{array}{l}\text { RMSEA } \\
\text { [I.C. } 90 \%]\end{array}$ & $\begin{array}{c}0,09 \\
{[0,08-0,10]}\end{array}$ & $\begin{array}{c}0,09 \\
{[0,08-0,10]}\end{array}$ & $\begin{array}{c}0,10 \\
{[0,09-0,11]}\end{array}$ & $\begin{array}{c}0,08 \\
{[0,07-0,10]}\end{array}$ \\
\hline TLI & 0,79 & 0,75 & 0,70 & 0,89 \\
\hline AGFI & 0,74 & 0,73 & 0,73 & 0,90 \\
\hline CFI & 0,83 & 0,80 & 0,73 & 0,91 \\
\hline AIC & 931,90 & 802,33 & 957,25 & 245,46 \\
\hline $\mathrm{BIC}$ & 1106,97 & 985,35 & 1128,34 & 356,87 \\
\hline MECVI & 2,38 & 2,05 & 2,44 & 0,62 \\
\hline
\end{tabular}

Nota: $\mathrm{X} 2$ = Qui-Quadrado; $\mathrm{gl}$ = graus de liberdade; $\mathrm{X} 2 / \mathrm{gl}=$ Qui-Quadrado normalizado; $\mathrm{GFI}$ = Índice de Qualidade do Ajuste; RMSEA = Raiz do Erro Quadrático Médio de Aproximação; TLI = Índice Tucker-Lewis; NFI = Índice de Ajuste Normalizado; AGFI = Índice de Qualidade de Ajuste Calibrado e CFI = Índice de Ajuste Comparativo; AIC = Critério de Informação Akaike; BIC = Critério de Informação Bayes; MECVI = Índice de Validação Cruzada Esperada. *Modelo adotado com melhor ajustamento (M4). 
A adequação do modelo proposto para a versão brasileira do BNSSS também foi evidenciada na análise de invariância considerando o sexo, cujos resultados apontaram a invariância configuracional e métrica entre homens e mulheres. Isso prova que a escala é versátil para utilização em grupos de atletas do sexo masculino e feminino. Esses resultados confirmam os já disponíveis na literatura, suportando a validade do construto no que se refere à invariância por sexo (Adie et al., 2004; Vlachopoulos, 2008). Tal achado indica que apesar das inconsistências encontradas no modelo latente, a versão brasileira da BNSSS é versátil para utilização em grupos de atletas do sexo masculino e feminino, indicando que a estrutura proposta - mesmos itens sendo explicados pelas mesmas variáveis latentes - se mantém para os diferentes sexos (Brown, 2012) e que os resíduos dos itens são iguais para ambos os grupos (Kline, 2012).

No entanto, a invariância estrutural do modelo não foi encontrada, o que permite afirmar que os níveis de correlação dos fatores não são os mesmos para os diferentes grupos. É importante salientar que o teste da invariância estrutural não avalia a variabilidade nos parâmetros psicométricos do instrumento, não comprometendo, assim, a confiabilidade estrutural do modelo (Sass, 2011). Desse modo, estabelecer a equivalência da variância e covariância das variáveis latentes não é um pré-requisito para avaliar diferenças de médias (invariância) entre grupos (Schmitt \& Kuljanin, 2008).

Os coeficientes de consistência interna do presente estudo foram semelhantes aos reportados na validação original ( $\mathrm{Ng}$ et al., 2011), além de ir ao encontro dos reportados por validações de escalas para o mesmo construto em outros contextos (Vlachopoulos \& Michailidou, 2006; Wilson et al., 2006; Gillet et al., 2008; Domínguez et al., 2010). Os valores das correlações item-dimensão foram satisfatórios, evidenciando que os itens realmente avaliam o que é proposto pelas dimensões. Correlações item-dimensão acima de 0,70 são consideradas indicativos de uma estrutura muito bem definida, visto que o fator explica pelo menos $50 \%$ da variância do item (Hair et al., 2009). Contudo, os itens que foram excluídos do modelo da AFE foram os mesmos que apresentaram correlações item-dimensão baixos na análise preliminar, já sugerindo sua condição problemática.

Ao analisar a estabilidade temporal, verificou-se que os 12 itens e três dimensões da versão brasileira da BNSSS se mostraram satisfatoriamente correlacionados com seu próprio reteste (Nunnally \& Berstein, 1994). Tais achados foram semelhantes aos reportados na validação original (Ng et al., 2011), comprovando sua estabilidade temporal.
Como não existem outras validações transculturais da BNSSS, comparando os valores de correlação intraclasse com escalas que avaliam os mesmos construtos em outros contextos da atividade física, também foi possível evidenciar resultados semelhantes (Vlachopoulos \& Michailidou, 2006; Wilson et al., 2006; Gillet et al., 2008; Domínguez et al., 2010). Estes resultados reiteram a validade da TAD, indo ao encontro da vasta literatura de estudos e instrumentos desenvolvidos com base neste referencial teórico (Vallerand et al., 2008).

Os achados do presente estudo incluem limitações que requerem ao leitor interpretá-los com cautela. A primeira limitação está relacionada à distribuição geográfica da amostra, uma vez que todos os atletas eram do estado do Paraná. Contudo, todos os atletas participavam de competições de nível estadual ou nacional e eram oriundos de diferentes regiões do Brasil. Estudos futuros também devem abordar essas questões relativas à validação cruzada de forma que os resultados possam ser generalizados para outras amostras. As propriedades psicométricas da BNSSS se apresentaram na mesma direção da versão original e aos demais estudos de validação transcultural, apesar das inconsistências nos itens já citados, evidenciando a validação cruzada do construto entre as amostras e culturas, fortalecendo a sua consistência e confiabilidade. Contudo, novos estudos deverão ser conduzidos para verificar a consistência da estrutura proposta por esses resultados, e investigar com mais profundidade os problemas com os itens excluídos e a dimensão da autonomia. Em segundo lugar, nosso estudo aborda apenas a confiabilidade, validade de conteúdo, construto e temporal do instrumento. Novos estudos já em andamento proporcionarão indicadores de validade externa (concorrente, discriminante ou preditiva) do instrumento. Além disso, estudos de validação transcultural devem ser conduzidos, como o já realizado por Vlachopoulos et al. (2013) com a Basic Psychological Needs in Exercise Scale.

Por fim, os resultados obtidos com a BNSSS sugerem propriedades psicométricas aceitáveis para sua utilização em atletas brasileiros, na avaliação da Satisfação das Necessidades Básicas. Esses resultados avançam na literatura por proporcionar evidências da utilização de um instrumento que pode fornecer informações relevantes que auxiliem os profissionais da área da Psicologia do Esporte e os técnicos no desenvolvimento de experiências que favoreçam a autodeterminação ou suas consequências no contexto esportivo.

\section{REFERÊNCIAS}

Adie, J., Duda, J. L., \& Ntoumanis, N. (2008). Autonomy support, basic need satisfaction and the optimal functioning of adult male and female sport participants: A test of basic needs theory. Motivation and Emotion, 32, 189-199. https://doi. org/10.1007/s11031-008-9095-Z
Balbinotti, M. A. A., \& Barbosa, M. L. L. (2008). Análise da consistência interna e fatorial confirmatório do Imprafe-126 com praticantes de atividades físicas gaúchos. Psico-USF, 13, 1-12.

Bara Filho, F. M., Andrade, D., Miranda, R., Nuñez, J.L., MartínAlbó, J., \& Ribas, P.R. (2011). Preliminary validation of a 
Brazilian version of the Sport Motivation Scale. Universitas Psychologica, 10(2), 557-566.

Brown, T. A. (2012). Confirmatory factor analysis for applied research. Ney York: Guilford Press.

Cheung, G. W., \& Lau, R. S. (2008). Testing mediation and suppression effects of latent variables: Bootstrapping with structural equation models. Organizational Research Methods, 11(2), 296-325. https://doi.org/10.1177/1094428107300343

Deci, E. L., \& Ryan, R. M. (1985). Intrinsic motivation and selfdetermination in human behavior. Nova York: Plenum.

Deci, E. L., \& Ryan, R. M. (2008). Facilitating optimal motivation and psychological well-being across life's domains. Canadian Psychology, 49, 14-23. http://doi.org/10.1037/07085591.49.1.14.

Deci, E. L., \& Ryan, R. M. (2012). Self-determination theory. In P. A. M. Van Lange, A. W. Kruglanski, \& E. T. Higgins (Eds.), Handbook of theories of social psychology (Vol. 1, pp. 416-437). Thousand Oaks, CA: Sage.

Domínguez, E., Martín, P., Martín-Albo, J., Núñez, J. L., \& León, J. (2010). Translation and validation of the Spanish version of the "Échelle de satisfaction des besoins psychologiques" in the sports context. The Spanish Journal of Psychology, 13(2), 1010-1020.

Fernándes, H., \& Vasconcelos-Raposo, J. (2005). Continuum de auto-determinação: Validade para a sua aplicação no contexto desportivo. Estudos de Psicologia, 10(3), 385-395. http://doi. org/10.1590/S1413-294X2005000300007.

Fernándes, H., Vasconcelos-Raposo, J., Lázaro, J. P., \& Dósil, J. (2004). Validación y aplicación de modelos teóricos motivacionales en el contexto de la educación física. Cuadernos de Psicología del Deporte, 4(1-2), 67-89.

Fornell, C., \& Larcker, D. F. (1981). Evaluating structural equation models with unobservable variables and measurement error. Journal of Marketing Research, 18, 39-50.

Gillet, N., Rosnet, E., \& Vallerand, R. J. (2008). Dévelopment d'une échelle de satisfaction des besoins foundamentaux en context sportif. Canadian Journal of Behavioral Science, 40, 230-37. https://doi.org/10.1037/a0013201.

Hair, J., Black, W., Babin, B., Anderson, R., \& Tatham, R. (2009). Multivariate data analysis. New Jersey: Pearson Educational.

Hernández-Nieto, R. A. (2002). Contributions to statistical analysis. Mérida: Universidad de Los Andes.

Hodge, K., Lonsdale, C., \& Ng, J. Y. Y. (2008). Burnout in elite rugby: Relationships with basic psychological needs fulfillment. Journal of Sports Sciences, 26, 835-844. https:// doi.org/10.1080/02640410701784525.

Kline, R. B. (2012). Principles and practice of structural equation modeling. New York: The Guilford Press.

Marôco, J. (2010). Análise de equações estruturais: Fundamentos teóricos, software e aplicações. Pêro Pinheiro: Report Number.

Moutão, J. M. R. P., Cid, L., Leitão, J. C., \& Alves, J. (2012). Tradução e validação do Perceived Autonomy Support: Exercise Climate Questionnaire numa amostra de praticantes de exercício portugueses. Psicologia: Reflexão e Crítica, 25(4), 701-708. http://doi.org/10.1590/S0102-79722012000400009

Ng, J. Y. Y., Lonsdale, C., \& Hodge, K. (2011). The Basic Needs Satisfaction in Sport Scale (BNSSS): Instrument development and initial validity evidence. Psychology of Sport and Exercise, 12, 257-64. https://doi.org/10.1016/j.psychsport.2010.10.006

Nunnally, C., \& Berstein, I. H. (1994). Psychometric theory. New York:McGraw-Hill.

Pasquali, L. (2010). Instrumentação psicológica: Fundamentos e práticas. Porto Alegre: Artmed.

Reeve, J., Nix, G., \& Hamm, D. (2003). Testing models of the experience of self- determination in intrinsic motivation and the conundrum of choice. Journal of Educational Psychology, $95,375-392$.
Reinboth, M., Duda, J. L., \& Ntoumanis, N. (2004). Dimensions of coaching behavior, need satisfaction, and the psychological and physical welfare of young athletes. Motivation and Emotion, 28, 297-313.

Sass, D. A. (2011). Testing measurement invariance and comparing latent factor means within a confirmatory factor analysis framework. Journal of Psychoeducational Assessment, 29(4), 347-363. http://doi.org/10.1177/0734282911406661.

Schmitt, N., \& Kuljanin, G. (2008). Measurement invariance: Review of practice and implications. Human Resource Management Review, 18(4), 210-222. https://doi.org/10.1016/j. hrmr.2008.03.003

Silva, R. B., Matias, T. S., Viana, M. S., \& Andrade, A. (2012). Relação da prática de exercícios físicos e fatores associados às regulações motivacionais de adolescentes brasileiros. Motricidade, 8(2), 8-21. http://doi.org/10.6063/ motricidade.8(2).708.

Teixeira, P. J., Carraça, E. V., Markland, D., Silva, M. N., \& Ryan, R. M. (2012). Exercise, physical activity, and self-determination theory: A systematic review. The International Journal of Behavioral Nutrition and Physical Activity, 9, 78. http://doi. org/ 10.1186/1479-5868-9-78.

Vallerand, R. J., Pelletier, L. G., \& Koestner, R. (2008). Reflections on Self-Determination Theory. Canadian Psychology, 49(3), 257-262. https://doi.org/10.1037/a0012804.

Vissoci, J. R. N., Vieira, L. F., Oliveira, L. P., \& Vieira, J. L. L. (2008). Motivação e atributos morais no esporte. Revista de Educação Física/UEM, 19(2), 173-82.

Vlachopoulos, S. P. (2008). The Basic Psychological Needs in Exercise Scale: Measurement invariance over gender. Structural Equation Modeling, 15, 114-35. https://doi. org/10.1080/10705510701758398

Vlachopoulos, S. P., \& Michailidou, S. (2006). Development and initial validation of a measure of autonomy, competence, and relatedness in exercise: The Basic Psychological Needs in Exercise Scale. Measurement in Physical Education and Exercise Science, 10(3), 179-201.

Vlachopoulos, S, P., Hulya Asci, F., Cid, L., Ersoz, G., GonzálezCutre, D., Moreno-Murcia, J. A., \& Moutão, J. (2013). Cross-cultural Invariance of the Basic Psychological Needs in Exercise Scale and Latent Mean Differences Among Greek, Spanish, Portuguese, and Turkish Samples. Psychology of Sport and Exercise, 14(5), 622-663. https://doi.org/10.1016/j. psychsport.2013.03.002

Wilson, P. M., Rogers, W.T., Rodgers, W. M., \& Wild, T. C. (2006). The psychological need satisfaction in exercise scale. Journal of Sport \& Exercise Psychology, 28, 231-251. 